\title{
High rates of parasite recrudescence following intermittent preventive treatment with sulphadoxine-pyrimethamine during pregnancy in Benin
}

\author{
Azizath Moussiliou ${ }^{1,2,3}$, Yolande Sissinto-Savi De Tove ${ }^{3}$, Justin Doritchamou ${ }^{1,2,3}$, Adrian JF Luty 1,2, \\ Achille Massougbodji ${ }^{3}$, Michael Alifrangis ${ }^{4,5}$, Philippe Deloron ${ }^{1,2}$ and Nicaise Tuikue Ndam ${ }^{1,2,3^{*}}$
}

\begin{abstract}
Background: Despite widespread parasite resistance to sulphadoxine-pyrimethamine (SP) its use for intermittent preventative treatment during pregnancy remains the policy in Benin and throughout most of sub-Saharan Africa.

Methods: In a prospective study, 982 pregnant women were recruited in Benin and followed until delivery. The prevalence of point mutations in the pfdhfr and pfdhps genes associated with Plasmodium falciparum resistance to SP during consecutive antenatal visits was determined. Parasites clearance among women infected at SP intake was assessed by microscopy and PCR. Association between the persistence of parasites and malaria consequences, were investigated. Recurrent parasites were genotyped to identify recrudescences from re-infections.

Results: The prevalence of pfdhfr/pfdhps quadruple mutants (triple pfdhfr + single pfdhps) was consistently above $80 \%$ while quintuple and sextuple mutants remained low. Importantly the higly mutated parasites apparently never included the two key mutations, pfdhfr $164 \mathrm{~L}$ or pfdhps 540E. Based on PCR results, SP failed to clear existing parasitaemia in half (48\%) of the women who were infected at IPTp schedule. The frequency of recrudescence reached $76 \%$ after the second dose. Women with persistent parasitaemia had an increased prevalence of anaemia $(P=0.03)$.
\end{abstract}

Conclusion: The data presented here, highlight the inability of SP to ensure optimal antiplasmodial protection in late pregnancy, and invite urgent consideration of an alternative drug or strategy.

Keywords: Plasmodium falciparum, Pregnancy, IPTp, Sulphadoxine-pyrimethamine, Mutations, pfdhfr, pfdhps, Benin

\section{Background}

Malaria is an important cause of maternal and perinatal morbidity and mortality [1]. Pregnancyassociated malaria (PAM) is characterized by the accumulation of Plasmodium falciparum-infected erythrocytes (PfIE) in placental intervillous spaces [2]. The occurrence of low birth weight (LBW) is one of the main consequences of PAM and this is associated with a

\footnotetext{
* Correspondence: nicaise.ndam@ird.fr

'Institut de Recherche pour le Développement, UMR216 Mère et enfant face aux infections tropicales, Faculté des sciences biologiques et

pharmaceutiques, 4, avenue de l'observatoire, Paris 75006, France

${ }^{2}$ Faculté de Pharmacie, Université Paris Descartes, PRES Sorbonne Paris Cité,

Paris 75270, France

Full list of author information is available at the end of the article
}

high risk of neonatal mortality. Approximately 100,000 children die annually in sub-Saharan Africa due to malaria-related LBW [3].

In Benin, anti-malarial treatment policy has long been based on the use of chloroquine (CQ) as first-line and $\mathrm{SP}$ as second-line. CQ was also used in prophylaxis of malaria during pregnancy. However, decreased therapeutic efficacies of both CQ and SP prompted the change of the national anti-malarial drug policy in 2004 by the official withdrawal of CQ and SP in the treatment of uncomplicated malaria. As reported in many parts of the world sulphadoxine-pyrimethamine (SP) has been 
used extensively in the past for treatment and prophylaxis of falciparum malaria [4]. One of the consequences is that the utility of SP in the treatment of malaria in sub-Saharan Africa has declined drastically in the last decade because of the emergence and spread of drug resistance of $P$. falciparum. However, SP is still used for malaria prevention during pregnancy as intermittent preventive treatment in pregnancy (IPTp). The SP-IPTp regimen has been recommended by the World Health Organization (WHO) [5] since 2004, and was adopted by Benin the same year. SP-IPTp regimen comprises the administration of at least two curative doses of SP starting in the second trimester of pregnancy (16 weeks of gestation) and ensuring a dose spacing interval of at least one month. SP continues to be the drug of choice for IPTp both because it is safe, easy to administer, and because a single treatment dose has long-lasting prophylactic effect (up to 60 days) [6,7]. SP efficacy is dependent on the number of mutations accumulated in the genes encoding the $P$. falciparum enzymes dihydrofolate reductase (DHFR) and dihydropteroate synthase (DHPS) [8,9]. Single nucleotide polymorphisms (SNPs) in the pfdhfr gene, at codons 51, 59, 108, and 164, and in the pfdhps gene, at codons 436, 437, 540,581 , and 613 are associated with in vitro resistance to pyrimethamine and sulphadoxine, respectively [10-12]. Parasite resistance to SP in vivo is largely associated with a triple mutation in pfdhfr (resulting in amino acid changes; N51I, C59R and S108N) coupled with a double mutation in pfdhps (A437G, K540E) [13-15]. Such P. falciparum parasites carrying quintuple mutations (triple pfdhfr with double pfdhps mutations) are highly prevalent in East Africa [16,17], but rare in West Africa [18], including Benin where a study from 2003 to 2005 showed that the most prevalent haplotype (85\%) was the quadruple (triple $p f d h f r$, single $p f d h p s$ ) mutant [19]. Although SP shows poor efficacy in children infected with quintuple mutant parasites, IPTp with SP seems to remain effective in preventing the adverse consequences of malaria on maternal and foetal outcomes in areas where a high proportion of $P$. falciparum parasites carry these quintuple mutations $[20,21]$.

From 2005 to 2007, a study in southern Benin [22] showed that the proportion of parasites carrying quadruple pfdhfr/pfdhps mutations during pregnancy was constantly above $80 \%$ whilst no quintuple mutants were detected. Recently, a prospective cohort study (STOPPAM) on $\sim 1,000$ pregnant women in southwestern Benin, four years after the change of malaria treatment policy in Benin was conducted. A subset of samples collected during the follow up were used to assess the prevalence and possible selection of molecular markers of SP resistance in the pfdhfr and pfdhps genes present at different gestational ages, and to characterize recurrent infections in the context of SP-IPTp.

\section{Methods}

\section{Study area}

The study was conducted from 2008 to 2010 in Comé, a semi-rural area of southern Benin, $70 \mathrm{~km}$ west of Cotonou, the capital of Benin. Malaria transmission is perennial, with two peaks during the two rainy seasons. The entomological inoculation rate (EIR) ranges from 35 to 60 infective bites per person and per year [23], with $P$. falciparum predominating [24]. The study area has been described elsewhere [25].

\section{Study design, collection and handling of blood samples}

The study comprised a cohort of pregnant women included before 24 weeks of pregnancy. Detailed descriptions of the pregnant women follow-up have been reported elsewhere [25]. Briefly, 982 women were recruited during the first antenatal visit and followed up monthly from inclusion to delivery. Two doses of SP-IPTp (IDA, The Netherlands) were administered following national guidelines. The exact gestational age was determined by using an ultrasound scans, performed with a portable ultrasound system (Titan Sonosite Bothell WA). A rapid diagnostic test (Parascreen ${ }^{\mathrm{Tn}}$, Zephyr Biomedicals Goa, India) for identification of $P$. falciparum infection was performed on capillary blood. Venous blood was taken at inclusion, at each antenatal visit, and at unscheduled 'emergency' visits when women presented at the clinic for health reasons. At delivery, placental and peripheral blood was also collected. Thick and thin blood films were prepared from all blood samples to confirm active P. falciparum infection, Giemsa-stained, and read by two independent, experienced microscopists.

Four separate drops of blood were spotted onto Whatman $3 \mathrm{MM}$ filter paper and stored with silica gel before DNA extraction using the Chelex 100 resin method [26]. Samples from 212 women were used for pfdhfr/pfdhps genotyping. A subset of 107 samples based on the availability of samples from days 7 to 60 following the SP uptake was identified to assess drug efficacy. Isolates from parasitized women at each SP-IPTp administration (either first or second dose) and each sample of these women until next SP administration, or for a 60day period were examined. Women having received anti-malarial treatment other than SP-IPTp during this follow up were excluded.

The study was approved by the ethics committees of the Research Institute for Development (IRD) in France and the Faculty of Health Science (University of Abomey-Calavi) in Benin. Written informed consent was given by all women participating in this study.

\section{Real-time PCR assay for the detection of Plasmodium falciparum infections}

A duplex real-time PCR assay using genus-specific and species-specific primers and probes (Plasmodium spp/P. 
falciparum) for the gene encoding the small subunit (18S) of Plasmodium rRNA was used to screen samples containing parasites following treatment, as described [27]. Samples underwent 40 cycles of amplification using the $\mathrm{ViiA}^{\mathrm{m}} 7$ Real-Time PCR system (Applied Biosystem) and were quantified using a DNA standard range made from a suspension of in vitro cultured 3D7 P. falciparum line (obtained through the MR4 as part of the BEI Resources Repository, NIAID, NIH: Plasmodium falciparum 3D7 GL, MRA-1001, deposited by Megan Dowler, Walter Reed Army Institute of Research).

\section{Nested PCR amplification of pfdhfr and pfdhps genes and detection of SNPs}

Parasite DNA was amplified with outer and nested specific primers targeting the pfdhfr and pfdhps genes, as described $[28,29]$. The SNPs in the pfdhfr and pfdhps genes were captured using sequence specific oligonucleotide probes-enzyme linked immunosorbent assay (SSOP-ELISA) technique, as described [29]. Briefly, 96well PCR plates (Maxisorp, Nunc, Roskilde, Denmark) were coated with streptavidin and incubated overnight at $+4^{\circ} \mathrm{C}$. Nested PCR products were diluted (1:10), denatured at $95^{\circ} \mathrm{C}$ and immediately cooled at $+4^{\circ} \mathrm{C}$ before being incubated on the plate. Individual digoxigeninconjugated SSOPs probes targeting pfdhfr codons c50/51 (CI/CN), c59 (C/R), c108 (S/N/T) and c164 (I/L), and pfdhps codons c436/c437 (AA/AG/SA/SG/FG), c540 (K/ $\mathrm{E})$, c581 (A/G) and c613 (A/S) were added. Plates were incubated in a shaker-incubator (ES-20/60, Biosan, Latvia) followed by stringent washing with tetra-methylammonium chloride (Sigma, Germany). Horseradish peroxidase-conjugated anti-digoxigenin antibody with ophenylene-diamine tablets dissolved in hydrogen peroxide was used as substrate. The reaction was stopped by addition of sulphuric acid. Optical densities (OD) were read at $492 \mathrm{~nm}$. For each SNP, samples were categorized into single or mixed infections. Single genotype infections harboured a single SNP present at OD values above the threshold of positivity. Mixed genotype samples contained a main SNP genotype and a minor genotype whose OD value was less than half (mixed with dominant genotype) or more than half (mixed with no dominant genotype) the OD value of the main genotype. For samples that contained infections categorized as single or mixed with a dominant SNP type at all analysed codons, results were combined to construct haplotypes.

\section{Molecular genotyping of the polymorphic genes $m s p 1$ (block 2) and $m s p 2$}

Analysis of MSP-2 (3D7 and FC27 allelic families) and MSP1 block 2 (K1, MAD20 and RO33 allelic families) of $P$. falciparum were sequentially performed by nested PCR in accordance with the genotyping protocol of
Snounou [30]. DNA genotypes of samples collected at baseline (IPTp uptake) and on the day of recurrent infection were compared according to band size and number, for each of the allelic families of msp 1 block 2 and $m s p 2$. To differentiate new infections from recrudescent ones, gel photographs were scored by visual comparison of DNA fragments obtained from baseline and reinfection samples. Unamplified samples and those showing the same $m s p 2$ profile on both consecutive samples were analysed on the msp 1 block 2 markers. An infection was considered to be a recrudescence when identical pre-treatment allele(s) were found in post-treatment samples, the two patterns being either completely identical or containing some missing or additional clones. Infections were considered as re-infections if the allelic pattern for any of the loci differed completely between the pre- and post-treatment samples.

\section{Data analysis}

Data were analysed using STATA version 11 (Stata Corp, College Station, TX, USA). Descriptive analyses were done by determining means, medians, standard deviations and interquartile ranges (IQR). The frequency of a particular mutant or haplotype was calculated as the proportion of the specific mutant or haplotype among the total number of samples successfully analysed for this mutation. Categorical variables were compared using the Pearson chi-square or Fisher exact test, while continuous variables were compared by the KruskallWallis test. The significance level $(P=0.05)$ was used in all analyses.

\section{Results}

In the STOPPAM study, a cohort of 982 women was enrolled and followed up, of whom 845 received two doses of SP, while 16, who were HIV-infected, received three doses. The first dose of SP-IPTp was given on average at 20 weeks of gestation, with the mean interval between the two doses of 35.4 days $(\mathrm{SD}=9.9)$. A total of 836 women gave birth to live newborn and successfully completed the follow up. The parasite prevalence based on microscopical examination of blood smears was $17.0 \%$ at enrolment, falling to $4.1 \%$ one month after the first dose of SP-IPTp, and remaining stable until one month after the second SP-IPTp administration, before rising to $11.1 \%$ at delivery. Infections were mostly polyclonal with a multiplicity of infection (MOI) [IQR] of 3.6 [2.2-4.8]. The MOI at IPTp schedules was higher than that of recurrent infections following treatment (4.5 vs 2.7, $P=0.04$ ). However no difference was seen in the MOI following the first or second dose $(P=0.6)$. Of the 191 women who were parasitized at baseline, 107 fulfilled the criteria to assess the in vivo drug efficacy (Figure 1). 
Trends of pfdhfr and pfdhps haplotypes throughout the antenatal clinic visits

The prevalence of point mutations in the pfdhfr and pfdhps genes was determined in samples collected from 212 HIV-negative women; their general characteristics are shown in Table 1 . At early pregnancy which corresponds to the day of the enrolment in the study, $88.0 \%$ of isolates consisted of triple mutant pfdhfr haplotypes CIRNI (C50, N51I, C59R, S108N and I164), 8.4\% were double mutant haplotypes (CICNI and CNRNI), while $3.6 \%$ were wild-type CNCSI (Figures 2A and 2B, Additional file 1: Table S1). This trend remained similar throughout the study period, as the majority of isolates $(>80 \%)$ were of the triple mutant pfdhfr CIRNI haplotype, and there was no difference in prevalence according to time of sampling $(P=0.7)$. For the $p f d h p s$ gene, $81.7 \%$ of isolates at early pregnancy were single mutants SGKAA, $11.6 \%$ were double mutant AGKAA (8.3\%) or SGKAS $(3.3 \%)$, and $6.7 \%$ were of the wild-type haplotype SAKAA. As for $p f d h f r$, the haplotype distribution remained similar throughout the follow up, although haplotypes such as AGKAS and AGKGS were only found late in the follow up, at a prevalence of 6-7\%. At codons pfdhfr I164 and pfdhps K540 only wild types were found. As expected, combining the two loci revealed that more than $80 \%$ of the parasite isolates were of the quadruple $p f d h f r / p f d h p s$ haplotypes (CIRNISGKAA and CIRNI-AGKAA) (Figure 2C). Although in low proportions $(<10 \%)$, some less-mutated haplotypes, as well as more highly mutated quintuple or sextuple allelic haplotypes, were observed, with a tendency for

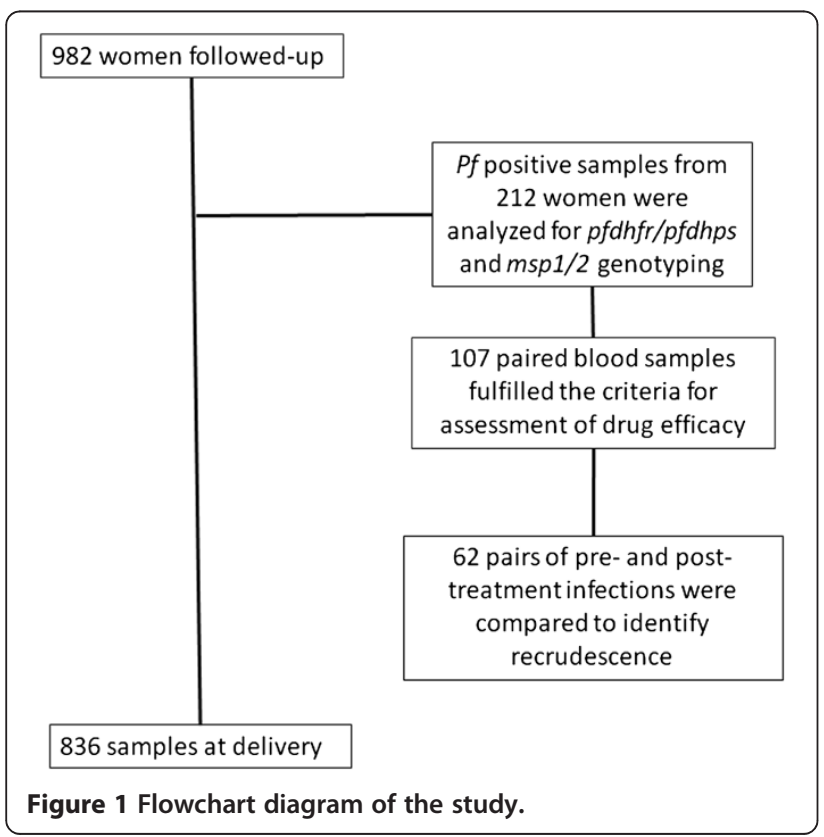

more quintuple and sextuple haplotypes in late pregnancy $(P=0.06)$ (Figure $2 C)$. The quintuple and sextuple mutants identified were mainly due to the combination of triple CIRNI $p f d h f r$ mutant haplotype with the double (SGKAS, AGKAS) and triple (AGKGS) mutants of pfdhps, respectively.

\section{Relationship between pfdhfr and pfdhps haplotypes and clinical outcome}

There was no significant difference in parasite density among women infected with a particular parasite haplotype at any stage of pregnancy: 872 parasites/ $\mu$ l $(105$ quadruple infections) vs 4,625 parasites/ $\mu \mathrm{l}$ (10 quintuple + sextuple infections, $P=0.2$ ). In addition, the distribution of the various haplotypes detected during pregnancy and at delivery was not associated with either risk of placental infection $(P=0.9)$, maternal anaemia $(P=0.5)$, or low birth weight $(P=0.6)$. Maternal anaemia was defined as haemoglobin concentration under $11 \mathrm{~g} / \mathrm{dL}$ and LBW as a birth weight $<2,500 \mathrm{~g}$.

\section{Identification and analysis of recurrent infections following IPTp uptake}

A total of 107 paired blood samples from women who had a positive blood smear at SP-IPTp administration (either first or second dose), and a blood sample available within a 60-day period following SP treatment were analysed by real-time PCR. Given the national guidelines of administration of two doses of SP-IPTp at an interval of at least one month, the follow-up time after the first dose of SP-IPT (29 days, range: 7-42) proved generally shorter than that after the second dose (37 days, range: $13-60, P=0.007)$. Parasites were detected in 37 women $(34.5 \%)$ by microscopy (blood smear $=\mathrm{BS}$ ) and in 63 (59.0\%) by PCR (Figure 3A, pooled data), of which approximately one third occurred in the first month after treatment (11\% for BS and 19\% for PCR), and two-thirds in the second month.

Segregating PCR-based data according to the sequence of the received dose of IPTp showed that the frequency of recurrence was higher after the second dose (64.6\%) than after the first one (51.5\%) (Figure 3B). Women were then split into those who cleared parasites during the follow up (BS-/PCR-), those in whom a submicroscopic parasitaemia persisted (BS-/PCR+) and those in whom a microscopic parasitaemia persisted $(\mathrm{BS}+/ \mathrm{PCR}+)$. No differential effect on birth weight was observed, but a trend for increased prevalence of maternal anaemia in women with persistent parasitaemia $(P=0.07)$ was noted. This trend was more explained by the extreme categories (BS-/PCR-) versus (BS+/PCR+) where maternal anaemia was observed in $52.5 \%$ vs $84.7 \%$, respectively $(P=0.03)$ (Additional file 2: Table S2). 
Table 1 Characteristics of the study population whose parasites were analysed by pfdhfr, pfdhps, msp 1 (block 2), and $m s p 2$ genotyping

\begin{tabular}{llll}
\hline & Mean (SD) & Median & Range \\
\hline $\begin{array}{l}\text { Mother's age in years } \\
(\mathbf{n}=\mathbf{2 1 2})^{*}\end{array}$ & $24.04(5.98)$ & 22 & $15-40$ \\
$\begin{array}{l}\text { Gestational age: weeks } \\
\text { of pregnancy }\end{array}$ & & & \\
$\quad \quad$ At inclusion $(n=83)$ & $18(4.1)$ & 17.4 & $4.0-25.0$ \\
$\quad$ At delivery $(n=88)$ & $39.0(1.9)$ & 39.1 & $33.4-42.3$ \\
$\begin{array}{l}\text { First dose SP-IPTp }{ }^{\S} \text { intake } \\
(\mathbf{n}=\mathbf{3 9})\end{array}$ & $20.9(3.2)$ & 21 & $15-28.4$ \\
$\begin{array}{l}\text { Second dose SP-IPTp intake } \\
(\mathbf{n}=\mathbf{5 7})\end{array}$ & $26.5(3.4)$ & 26.3 & $20-33.4$ \\
$\begin{array}{l}\text { Post second dose IPTp } \\
(\mathbf{n}=\mathbf{3 5})\end{array}$ & $30.5(3.5)$ & 31 & $20-35.9$ \\
$\begin{array}{l}\text { Newborn's birth weight in } \mathbf{g} \\
(\mathbf{n}=\mathbf{2 1 2})\end{array}$ & $2,881.2(488.2)$ & 2,900 & $1,100-4,050$ \\
\hline
\end{tabular}

Footnote: * Primigravidae $(n=63)$, Secundigravidae $(n=54)$,

Multigravidae $(\mathrm{n}=95)$.

${ }^{\S}$ SP-IPTP, Sulphadoxine-pyrimethamine used for intermittent preventive treatment during pregnancy.
Genotyping of recurrent infections following SP-IPTp uptake A total of 62 pairs of pre- and post-treatment infections identified by PCR were sequentially genotyped by amplifying the $m s p 2$ and $m s p 1$ (block 2 ) genes. Among these sample pairs, $52(84.0 \%)$ were successfully genotyped. The mean parasitaemia of recurrent samples among unresolved infections, as estimated by real-time PCR, was lower than in those successfully genotyped (40 vs 1,000 parasites $/ \mu \mathrm{L}, P<0.01$ ), explaining the failure to genotype some recurrences. Overall, recrudescent infections were more frequent than new infections (57.6\% vs 42.3\%). The frequency of recrudescence was markedly higher after the second SP-IPTp dose (75.9\% of re-infections) than after the first (34.8\%) (Figure 4).

The $p f d h f r / p f d h p s$ haplotype analysis of this sub-group of consecutively collected parasite isolates showed a doubling in the frequency of quintuple mutants (from 9.1 to $20.0 \%$ ) at the expense of quadruple mutants in post-treatment samples $(P=0.06)$, suggestive of a treatment-based selection effect (Figure 5).

\section{Discussion}

Historically, the SP drug combination has been widely used in malaria-endemic countries of sub-Saharan Africa
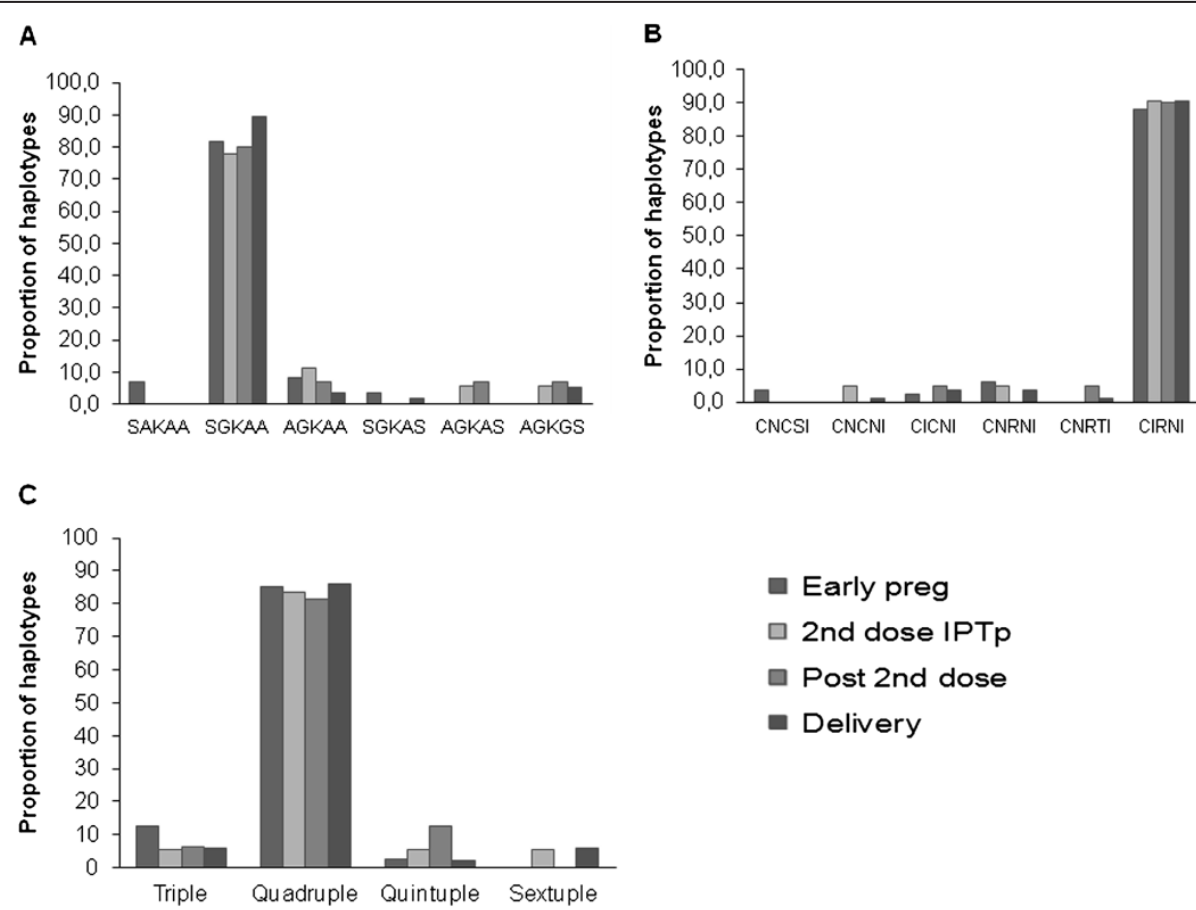

Figure 2 Proportions of SNPs associated with SP resistance. DNA from laboratory strains with known pfdhfr and pfdhps haplotypes (3D7, FRC3, K1, HB3, DD2, 7G8) were used as positive controls. Panel A: Proportion of SNPs associated with SP resistance on pfdhps gene: codons S436A, A437G, K540E, K613S/T and A581G. SGKAA, AGKAA are single mutant haplotypes while AGKGS represents double mutant. Mixed infections were excluded in the derivation of allelic haplotypes. Panel B: Proportions of SNPs associated with SP resistance on pfdhfr gene: codons N51I, C59R, S108N and I164L. Proportions are shown for isolates collected at different time-points. CICNI, CNRNI and CNRTI are double mutant haplotypes while CIRNI represents triple mutant. Panel C: Proportions of combined pfdhfr and pfdhps allelic haplotypes. The combined analysis of both loci revealed that more than $80 \%$ of the parasite isolates had quadruple pfdhfr/pfdhps allelic haplotypes. 

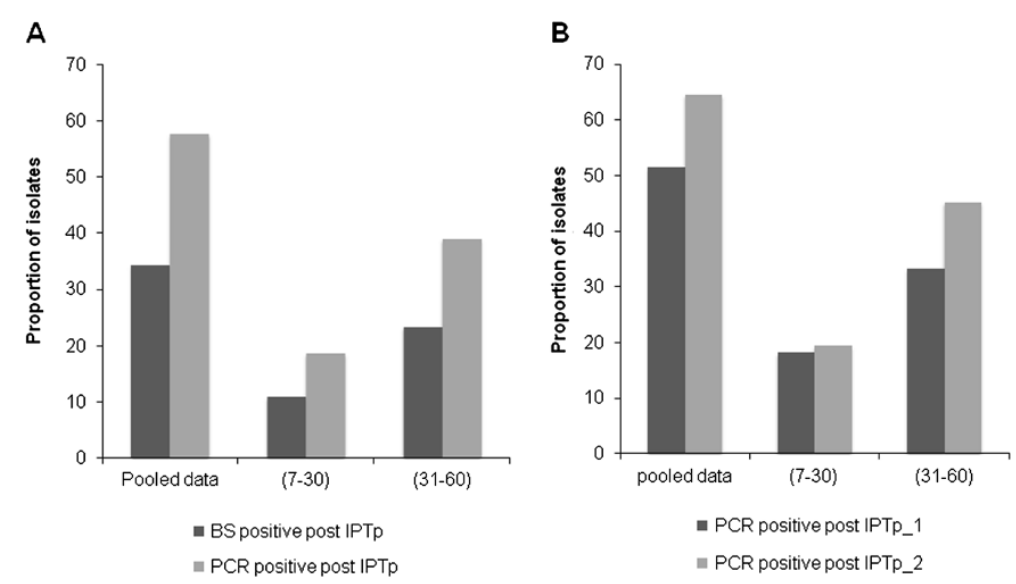

Figure 3 Prevalence rates of recurrent infections. Plasmodium parasites were counted against 200 leukocytes with a $100 \times$ objective lens under oil immersion. Each qPCR reaction mixture contained $5 \mu \mathrm{L}$ DNA template in a final volume of $20 \mu \mathrm{L}, 10 \mu \mathrm{L}$ of Master Mix (Applied Biosystem), both the genus-specific and P. falciparum specific primers and probes detection system (Plasmo/Pf) as described [24]. Panel A: Prevalence rates of recurrent infections assessed by microscopy (BS) and PCR. Data are shown as pooled (covering the two months following treatment) or split into first and second month (days 7-30 or 31-60). Panel B: Prevalence rates of recurrent infections assessed by PCR according to the IPTp dose received. Data are shown as pooled (covering the two months following treatment) or split into first and second month (days seven-30 or 31-60).

as a first-line drug to treat uncomplicated $P$. falciparum malaria. The emergence and widespread development of $P$. falciparum resistance to SP occurred over the last 30 years to reach the currently unacceptable level [31-37]. Characterization of the SNPs associated with SP resistance has provided an epidemiological tool to investigate the extent of resistance, as an alternative to the expensive and labour-intensive in vivo and in vitro drug sensitivity tests. Clearly, intensive past use of SP has been responsible for this spread of SP resistance, and to the upward trend in the prevalence rates of highly mutated pfdhfr/pfdhps parasites in West and East Africa $[36,38]$. The current study offered an excellent opportunity to investigate the level of penetration and the

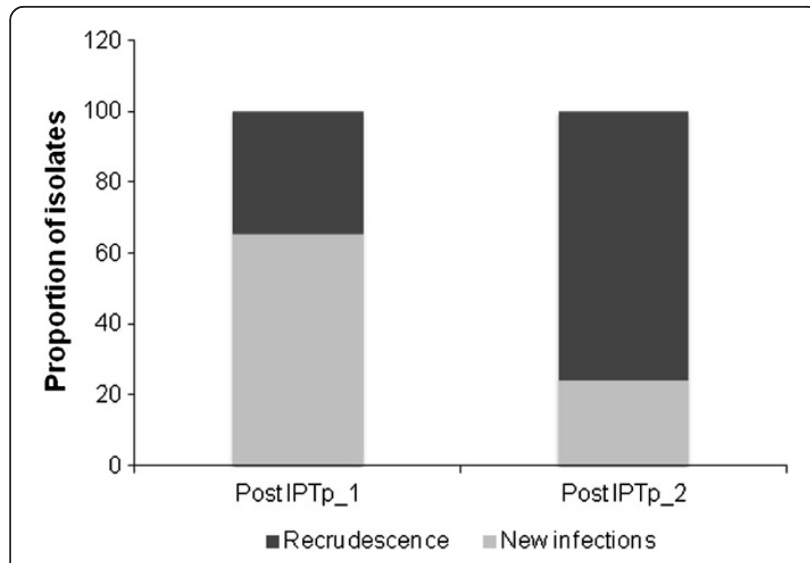

Figure 4 Proportions of recrudescent and new infections following SP treatment according to the sequence of IPTp uptake. dynamics of the critical SNPs associated with SP resistance in a longitudinal analysis of P. falciparum isolates collected from pregnant women using SP-IPTp in Benin.

Based on blood smear, the highest prevalence rate of P. falciparum infections (17\%) was observed at inclusion when women were screened for the first time, and had not yet received any anti-malarials, SP or other. Regardless of gestational age, the overwhelming majority of parasite isolates $(\sim 90 \%)$ carried the triple mutant $p f d h f r$ (CIRNI) haplotype in association with the single pfdhps (SGKAA) mutation. A similarly high prevalence of pfdhfr/pfdhps mutations (85\%) before any administration of SP-IPTp was reported in Southern Benin [22]. The current study provides an update of the situation six years after the withdrawal of SP as first-line anti-malarial drug, and three years after the study by Bertin [22], and

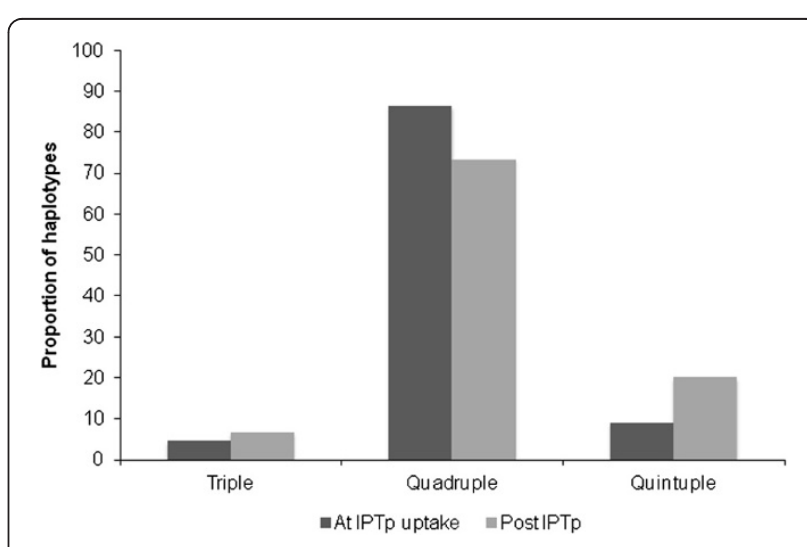

Figure 5 Prevalence of pfdhfr/pfdhps haplotypes in consecutive parasite samples at baseline and after IPTp uptake. 
suggests that the level of resistance has remained largely unchanged despite the reduced SP drug pressure on the parasite population. The identification of pfdhps codons 581 and 613 mutants is in agreement with published data, which indicates a comparatively low prevalence of these mutations [18]. Although, any significant relationship between the highly mutated haplotypes, such as the quintuple and sextuple mutants, with the occurrence of LBW and maternal anaemia was revealed, there is a tendency for the association of these mutants with higher peripheral parasite densities despite the limited sample size.

Of even greater concern, perhaps, is the fact that SPIPTp failed to clear almost half of the infections overall, and that a large proportion of those occurred after completion of the second dose of SP-IPTp. In this study, the parasitological failure rate of SP, when analysed within a month, was 11 and $19 \%$ as determined by microscopy and PCR, respectively (Figure 2A). This is of course far lower than the failure rate of $73 \%$ reported among children less than five years old in the same region of Benin with a 28-day follow up [39], but entirely consistent with that reported in Tanzania where the failure rate at 28 days after SP treatment was 16\% among pregnant women versus $80 \%$ in under five-year old children [40]. The substantially lower failure rates in pregnant women than in children is attributable to the stronger acquired anti-malarial immunity in adults, as well as possible pregnancy-specific differences in pharmacokinetics [41]. The extended follow up (60-day) in this study allowed detection of a greater number of recurrences, reaching $66 \%$ by PCR after the second dose of SP-IPTp. Clearly a longer follow up equates to less circulating drug and more exposure to re-infections. The longer follow up nevertheless also increased the ability to detect true recrudescence. Up to $76 \%$ of recurrent infections corresponded to true recrudescence. The fact that most of these infections were submicroscopic suggests that SP significantly reduced parasite densities, without complete clearance. The increasing parasite prevalence observed in the cohort during the third trimester, several weeks after the last SP-IPTp dose, is likely to be related to SP's lack of efficacy. The effects of such submicroscopic infections at various gestational ages on the health of mother and foetus remain unknown. In this study, a clear relationship with birth weight, probably due to low sample size was unable to be demonstrated, but a trend towards more maternal anaemia was observed. A recent WHO review [21] concluded that, despite the high proportion of $P$. falciparum parasites carrying quintuple mutations, associated with in vivo SP resistance in some African countries, SPIPTp remains effective in preventing the adverse consequences of malaria on maternal and foetal outcomes.
A previously report showed that the incidence of malaria attacks, i e, symptomatic episodes, in the entire STOPPAM cohort reached a peak in the third trimester, long after the intake of the second dose of SP-IPTp [25]. This observation, allied to the current findings, indicates that the completion of the two-dose SP-IPTp regimen at a comparatively early gestational age left women susceptible to (re-)infection before delivery. Despite a decline from 17 to $4 \%$ in the prevalence of peripheral parasitaemia after treatment, the occurrence of recrudescent infections contributes to the rise in the prevalence later in pregnancy, as evidenced by the $11 \%$ prevalence found at delivery.

\section{Conclusions}

These findings thus lend weight to the previous argument [42] for modification of the current SP-IPTp strategy to three doses instead of two in all mothers, as a third dose may contribute to maintaining low parasite densities and subdue adverse clinical outcomes. This is in line with a recently published meta-analysis from seven trials of 6,281 pregnancies in Africa, showing that the addition of a third SP dose was associated with an increase of birth weight and a lower risk of LBW than the standard two-dose regimen [43].

However, the high rate of recrudescence observed here highlights the lack of efficacy of SP in pregnant women. Under such conditions, changing the IPTp regimen using SP may seem to be based on pragmatic observations, but will only shift the problem as SP resistance will increase further. The urgency remains to find a more suitable alternative drug or a vaccine.

\section{Additional files}

Additional file 1: Table S1. Constructed haplotypes of pfdhfr and pfdhps detected during the follow-up.

Additional file 2: Table S2. Clinical comparisons between women with and without persisting parasitaemia.

\section{Abbreviations \\ PAM: Pregnancy-associated malaria; PfIE: Plasmodium falciparum-infected erythrocytes; LBW: Low birth weight; SP: Sulphadoxine-pyrimethamine; IPTp: Intermittent preventive treatment; SNPS: Single nucleotide polymorphisms; DHFR: Dihydrofolate reductase; DHPS: Dihydropteroate syntase; OD: Optical density; MOl: Multiplicity of infection.}

\section{Competing interests}

The authors declare that they have no competing interests.

\section{Authors' contributions}

PD and NTN conceived and designed the study. AMO, YSSDT and JD performed the laboratory experiments. AMO, MA and NTN analysed the data. AMA contributed reagents/materials/analysis tools. AMO, JD, MA, AJFL, PD and NTN drafted and finalized the manuscript. The final manuscript was read and approved by all authors. 


\section{Acknowledgements}

We are grateful to all the women who participated in the study, particularly to those involved in STOPPAM project. We thank all the medical staffs of Akodeha, Come Central and Ouedeme Pedah health centres, for their valuable contributions. We also thank Nadine Fievet for facilitation of the field work, Parfait Houngbegnon for help in data analysis and Jerome Clain for critical review of the manuscript.

\section{Author details}

${ }^{1}$ Institut de Recherche pour le Développement, UMR216 Mère et enfant face aux infections tropicales, Faculté des sciences biologiques et pharmaceutiques, 4, avenue de l'observatoire, Paris 75006, France. ${ }^{2}$ Faculté de Pharmacie, Université Paris Descartes, PRES Sorbonne Paris Cité, Paris 75270, France. ${ }^{3}$ Centre d'Etude et de Recherche sur le Paludisme associé à la Grossesse et à l'Enfance, Faculté des Science de Santé, Université d'Abomey-Calavi, Cotonou 01 BP 188, Benin. ${ }^{4}$ Department of Internationa Health, Immunology and Microbiology, Centre for Medical Parasitology Faculty of Health, Medical Sciences, University of Copenhagen, Copenhagen K 1014, Denmark. ${ }^{5}$ Department of Infectious Diseases, Copenhagen University Hospital (Rigshospitalet), Copenhagen K 1014, Denmark.

Received: 8 April 2013 Accepted: 4 June 2013

Published: 10 June 2013

\section{References}

1. Brabin BJ: An analysis of malaria in pregnancy in Africa. Bull World Health Organ 1983, 61:1005-1016.

2. Fried M, Duffy PE: Adherence of Plasmodium falciparum to chondroitin sulfate $a$ in the human placenta. Science 1996, 272:1502-1504.

3. Desai M, TerKuile FO, Nosten F, McGready R, Asamoa K, Brabin B, Newman RD: Epidemiology and burden of malaria in pregnancy. Lancet Infect Dis 2007, 7:93-104.

4. McGready R, Nosten F: Proxies and prevention of malaria in pregnancy. Lancet Infect Dis 2012, 12:902-903.

5. WHO: A strategic framework for malaria prevention and control during pregnancy in the Africa region. Brazzaville, Congo: WHO Regional Office for Africa; 2004. AFR/MAL/04/01.

6. Watkins WM, Mberu EK, Winstanley PA, Plowe CV: The efficacy of antifolate antimalarial combinations in Africa: a predictive model based on pharmacodynamic and pharmacokinetic analyses. Parasitol Today 1997, 13:459-464.

7. Watkins WM, Mberu EK, Winstanley PA, Plowe CV: More on 'the efficacy of antifolate antimalarial combinations in Africa'. Parasitol Today 1999, 15:131-132.

8. Peterson DS, Walliker D, Wellems TE: Evidence that a point mutation in dihydrofolate reductase-thymidylate synthase confers resistance to pyrimethamine in falciparum malaria. Proc Natl Acad Sci U S A 1988, 85:9114-9118.

9. Triglia T, Menting JGT, Wilson C, Cowman AF: Mutations in dihydropteroate synthase are responsible for sulfone and sulfonamide resistance in Plasmodium falciparum. Proc Natl Acad Sci U S A 1997, 94:13944-13949.

10. Cortese JF, Plowe CV: Antifolate resistance due to new and known Plasmodium falciparum dihydrofolate reductase mutations expressed in yeast. Mol Biochem Parasitol 1998, 94:205-214

11. Gregson A, Plowe CV: Mechanisms of resistance of malaria parasites to antifolates. Pharmacol Rev 2005, 57:117-145.

12. Plowe CV, Kublin JG, Doumbo OK: P. falciparum dihydrofolate reductase and dihydropteroatesynthase mutations: epidemiology and role in clinical resistance to antifolates. Drug Resist Updat 1998, 1:389-396.

13. Kublin JG, Dzinjalamala FK, Kamwendo DD, Malkin EM, Cortese JF, Martino LM, Mukadam RA, Rogerson SJ, Lescano AG, Molyneux ME, Winstanley PA, Chimpeni P, Taylor TE, Plowe CV: Molecular markers for failure of sulfadoxine-pyrimethamine and chlorproguanil-dapsone treatment of Plasmodium falciparum malaria. J Infect Dis 2002, 185:380-388

14. Kyabayinze D, Cattamanchi A, Kamya MR, Rosenthal PJ, Dorsey G: Validation of a simplified method for using molecular markers to predict sulfadoxine-pyrimethamine treatment failure in African children with falciparum malaria. AmJTrop Med Hyg 2003, 69:247-252.

15. Omar SA, Adagu IS, Warhurst DC: Can pretreatment screening for dhps and dhfr point mutations in Plasmodium falciparum infections be used to predict sulfadoxine-pyrimethamine treatment failure? Trans $R$ Soc Trop Med Hyg 2001, 95:315-319.

16. Harrington WE, Mutabingwa TK, Kabyemela E, Fried M, Duffy PE: Intermittent treatment to prevent pregnancy malaria does not confe benefit in an area of widespread drug resistance. Clin Infect Dis 2011, 53:224-230.

17. Daniel TR M, Schmiegelow C, Mmbando B, Boström S, Oesterholt M Magistrado P, Perhson C, John D, Salanti A, Luty AJF, Lemnge M, Theander T, Alifrangis M, Lusingu J: Infections with Plasmodium falciparum sextuple Pfdhfr/Pfdhps allelic haplotypes during pregnancy is associated with decreased birth weight in Korogwe, Tanzania. Emer Inf Dis. In press.

18. Drug resistance maps. www.drugresistancemaps.org.

19. Nahum A, Erhart A, Ahounou D, Bonou D, Van Overmeir C, Menten J, Akogbeto M, Coosemans M, Massougbodji A, D'Alessandro U: Extended high efficacy of the combination sulphadoxine-pyrimethamine with artesunate in children with uncomplicated falciparum malaria on the Benin coast. West Africa. Malar J 2009, 8:37.

20. Leke RG, Taylor DW: The use of intermittent preventive treatment with sulfadoxine-pyrimethamine for preventing malaria in pregnant women Clin Infect Dis 2011, 53:231-233.

21. World Health Organization: Evidence Review Group: Intermittent Preventive Treatment of malaria in pregnancy (IPTp) with Sulfadoxine- Pyrimethamine (SP). Geneva: WHO; 2012. http://www.who.int/malaria/mpac/sep2012/ iptp_sp_erg_meeting_report_july2012.pdf

22. Bertin G, Briand V, Bonaventure D, Carrieu A, Massougbodji A, Cot M, Deloron P: Molecular markers of resistance to sulphadoxinepyrimethamine during intermittent preventive treatment of pregnant women in Benin. Malar J 2011, 10:196.

23. Kelly-Hope LA, McKenzie FE: The multiplicity of malaria transmission: a review of entomological inoculation rate measurements and methods across sub-Saharan Africa. Malar J 2009, 8:19.

24. Sandeu M, Moussiliou A, Moiroux N, Padonou G, Massougbodji A, Corbel V, Ndam NT: A multiplex real-time PCR assay for Plasmodium parasites panspecies and species-specific detection and quantification in malaria vectors. PLoS One 2012, 7:e52719.

25. Huynh BT, Fievet N, Gbaguidi G, Dechavanne S, Borgella S, Guézo-Mévo B, Massougbodji A, Ndam NT, Deloron P, Cot M: Influence of the timing of malaria infection during pregnancy on birth weight and on maternal anemia in Benin. AmJTrop Med Hyg 2011, 85:214-220.

26. Wooden J, Kyes S, Sibley CH: PCR and strain identification in plasmodium falciparum. Parasitol Today 1993, 9:303-305.

27. Diallo A, Ndam NT, Moussiliou A, Dos Santos S, Ndonky A, Borderon M, Oliveau S, Lalou R, Le Hesran JY: Asymptomatic carriage of Plasmodium in urban Dakar: the risk of malaria should not be underestimated. PLoS One 2012, 7:e31100.

28. Pearce RJ, Drakeley C, Chandramohan D, Mosha F, Roper C: Molecular determination of point mutation haplotypes in the dihydrofolatereductase and dihydropteroatesynthase of Plasmodium falciparum in three districts of northern Tanzania. Antimicrob Agents Chemother 2003, 47:1347-1354.

29. Alifrangis M, Enosse S, Pearce R, Drakeley C, Roper C, Khalil IF, Nkya WM, Rønn AM, Theander TG, Bygbjerg IC: A simple, high-throughput method to detect Plasmodium falciparum single nucleotide polymorphisms in the dihydrofolatereductase, dihydropteroate synthase, and P. falciparum chloroquine resistance transporter genes using polymerase chain reaction- and enzyme-linked immunosorbent assay-based technology. AmJTrop Med Hyg 2005, 72:155-162

30. Snounou G, Zhu X, Siripoon N, Jarra W, Thaithong S, Brown KN, Viriyakosol S: Biased distribution of msp1 and msp2 allelic variants in Plasmodium falciparum populations in Thailand. Trans R Soc Trop Med Hyg 1999, 93:369-734

31. Maïga O, Djimdé AA, Hubert V, Renard E, Aubouy A, Kironde F, Nsimba B Koram K, Doumbo OK, Le Bras J, Clain J: A shared Asian origin of the triple-mutant dhfr allele in Plasmodium falciparum from sites across Africa. J Infect Dis 2007, 196:165-172.

32. Sridaran S, McClintock SK, Syphard LM, Herman KM, Barnwell JW, Udhayakumar $\mathrm{V}$ : Anti-folate drug resistance in Africa: meta-analysis of reported dihydrofolate reductase (dhfr) and dihydropteroate synthase (dhps) mutant genotype frequencies in African Plasmodium falciparum parasite populations. Malar J 2010, 9:247

33. Somé AF, Séré $Y Y$, Dokomajilar C, Zongo I, Rouamba N, Greenhouse B, Ouédraogo JB, Rosenthal PJ: Selection of known Plasmodium falciparum 
resistance-mediating polymorphisms by artemether-lumefantrine and amodiaquine-sulfadoxine-pyrimethamine but not dihydroartemisininpiperaquine in Burkina Faso. Antimicrob Agents Chemother 2010, 54:1949-1954.

34. Alam MT, De Souza DK, Vinayak S, Griffing SM, Poe AC, Duah NO, Ghansah A, Asamoa K, Slutsker L, Wilson MD, Barnwell JW, Udhayakumar V, Koram KA: Selective sweeps and genetic lineages of Plasmodium falciparum drug-resistant alleles in Ghana. J Infect Dis 2011, 203:220-227.

35. Malisa A, Pearce R, Abdullah S, Mutayoba B, Mshinda H, Kachur P, Bloland P, Roper C: Molecular monitoring of resistant dhfr and dhps allelic haplotypes in Morogoro and Mvomero districts in south eastern Tanzania. Afr Health Sci 2011, 11:142-150.

36. Duah NO, Quashie NB, Abuaku BK, Sebeny PJ, Kronmann KC, Koram KA: Surveillance of molecular markers of Plasmodium falciparum resistance to sulphadoxine-pyrimethamine 5 years after the change of malaria treatment policy in Ghana. AmJTrop Med Hyg 2012, 87:996-1003.

37. Lin JT, Mbewe B, Taylor SM, Luntamo M, Meshnick SR, Ashorn P: Increased prevalence of dhfr and dhps mutants at delivery in Malawian pregnant women receiving intermittent preventive treatment for malaria. Trop Med Int Health 2013, 18:175-178.

38. Iriemenam NC, Shah M, Gatei W, Van Eijk AM, Ayisi J, Kariuki S, Vanden Eng J, Owino SO, Lal AA, Omosun YO, Otieno K, Desai M, ter Kuile FO, Nahlen B, Moore J, Hamel MJ, Ouma P, Slutsker L, Shi YP: Temporal trends of sulphadoxine-pyrimethamine (SP) drug-resistance molecular markers in Plasmodium falciparum parasites from pregnant women in western Kenya. Malar J 2012, 11:134.

39. Faucher JF, Aubouy A, Adeothy A, Cottrell G, Doritchamou J, Gourmel B, Houzé P, Kossou H, Amedome H, Massougbodji A, Cot M, Deloron P: Comparison of sulfadoxine-pyrimethamine, unsupervised artemetherlumefantrine, and unsupervised artesunate-amodiaquine fixed-dose formulation for uncomplicated Plasmodium falciparum malaria in Benin: a randomized effectiveness noninferiority trial. J Infect Dis 2009, 200:57-65.

40. Mutabingwa TK, Muze K, Ord R, Briceño M, Greenwood BM, Drakeley C, Whitty CJ: Randomized trial of artesunate + amodiaquine, sulfadoxinepyrimethamine + amodiaquine, chlorproguanal-dapsone and SP for malaria in pregnancy in Tanzania. PLoS One 2009, 4:e5138.

41. Deloron P, Bertin G, Briand V, Massougbodji A, Cot M: Sulphadoxine/ pyrimethamine intermittent preventive treatment for malaria during pregnancy. Emerg Infect Dis 2010, 16:1666-1670.

42. Huynh BT, Fievet N, Briand V, Borgella S, Massougbodji A, Deloron P, Cot M: Consequences of gestational malaria on birth weight: finding the best timeframe for intermittent preventive treatment administration. PLoS One 2012, 7:e35342.

43. Kayentao K, Garner P, Van Eijk AM, Naidoo I, Roper C, Mulokozi A, MacArthur JR, Luntamo M, Ashorn P, Doumbo OK, Ter Kuile FO: Intermittent preventive therapy for malaria during pregnancy using 2 vs 3 or more doses of sulfadoxine-pyrimethamine and risk of low birth weight in Africa: systematic review and meta-analysis. JAMA 2013, 309:594-604.

doi:10.1186/1475-2875-12-195

Cite this article as: Moussiliou et al:: High rates of parasite recrudescence following intermittent preventive treatment with sulphadoxine-pyrimethamine during pregnancy in Benin. Malaria Journal 2013 12:195.

\section{Submit your next manuscript to BioMed Central and take full advantage of:}

- Convenient online submission

- Thorough peer review

- No space constraints or color figure charges

- Immediate publication on acceptance

- Inclusion in PubMed, CAS, Scopus and Google Scholar

- Research which is freely available for redistribution 